IAU Colloquium 164: Radio Emission from Galactic and Extragalactic Compact Sources

ASP Conference Series, Vol. 144, 1998

J. A. Zensus. G. B. Taylor, \& J.M. Wrobel (eds.)

\title{
Two Types of Jet in Cepheus A
}

\section{A. Hughes}

Department of Physics, Queen's University Kingston, Ontario K7L 3N6, Canada

Abstract. The young star forming region, Cephens A, has been monitored since 1981, and shows four new types of time-dependent events. Two of these are briefly described.

\section{Introduction}

Cepheus $A$ is a region where young stars are forming. When first observed with the VLA of the National Radio Astronomy Observatory, about 13 objects were detected (Hughes \& Wouterloot 1984; Hughes 1985). Most had a size of $1,000 \mathrm{AU}$, and it was not expected that they would last for very long. This led to a monitoring of the region which has continued for more than 10 years. The results have shown the presence of highly time dependent sources 8 and 9 (Hughes 1991), a jet which propagates to the SE with a cross speed of $\sim 300$ $\mathrm{km} \mathrm{s}^{-1}, 7(\mathrm{c})$ being the Mach shock and $7(\mathrm{~d})$ the jet shock (Hughes 1993), a new type of radio source, $3(\mathrm{a})$, which comprises two non-thermal sources diverging at a speed of $\sim 370 \mathrm{~km} \mathrm{~s}^{-1}$ (Hughes 1997), and 7(a) which contains three B3 stars in orbit (Hughes, in preparation). All these aspects are important since they deal with processes that take place at the very earliest times when the region is highly obscured. This paper deals with the jet propagating to the SE, and the two diverging non-thermal objects. Figure 1 shows an image of the region.

\section{The Jet to the SE, Sources $7(\mathrm{c})$ and $7(\mathrm{~d})$}

These two sources are non-thermal, and appear to be the Mach shock, $7(\mathrm{c})$, and jet shock, $7(\mathrm{~d}) .7(\mathrm{c})$ has a measured cross speed of $300 \mathrm{~km} \mathrm{~s}^{-1}$, and $7(\mathrm{~d})$ is assumed to have the same speed, but has too small a flux density for accurate determination of speed. Source $7(\mathrm{~d})$ has associated $\mathrm{H}_{2}$ and [Fe II] emission in the near IR (Goetz et al. 1995), which appears to be collisionally excited. The jet is likely young, $\sim 300$ years old, and seems to originate near to Sources 2 or 9.

\section{The Evolving Radio Source 3(a)}

Source 3(a) was clearly present in 1981, but then decreased in flux density at $6-\mathrm{cm}$, and appeared to widen at $20-\mathrm{cm}$. It was shown to consist of two sources which diverged at a cross speed of $370 \mathrm{~km} \mathrm{~s}^{-1}$. One component could be traced from JD 2445000 to JD 244800 , during which time it decreased in flux density from $2.5 \mathrm{mJy}$ to $0.25 \mathrm{mJy}$, but the spectral index changed from +0.2 to -1.20 over the period of $\sim 3,000$ days. A transient $\mathrm{H}_{2} \mathrm{O}$ maser was seen during the early stages (Cohen, Rowland, \& Blair 1984), indicating that the source was a protostar, but the event occurred $\sim 1,800$ days before monitoring began. The second component was not detected at $6-\mathrm{cm}$, showing evidence for a highly non- 


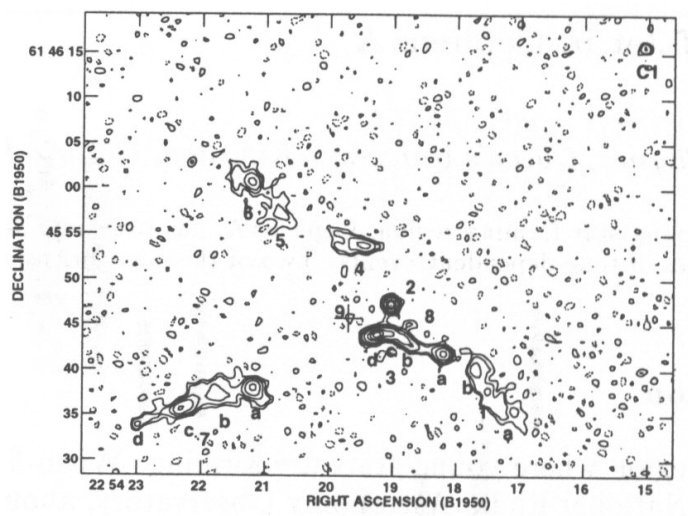

Figure 1. An image of Cepheus A obtained with the VLA in 1981, using the "B" configuration at $6-\mathrm{cm}$. It is included so as to show the positions of the various radio sources.

thermal source. It is suggested that the outbursts are likely to be jets associated with a protostar or PMS star ridding itself of its magnetic field.

Other sources are 8 and 9 , which have flux densities that are highly timedependent and are produced by a gyro-synchrotron mechanism, and 7(a) which contains an association of three B3 stars, and is more developed than the other objects discussed.

\section{Conclusion}

The monitoring program has shown that the taking of snap-shot observations of star forming regions does not tell the whole story. The present program has shown four new events that have timescales of 80-100 days for 8 and 9 , and $\sim$ 10-15 years for the others. It is believed that Sources $3(\mathrm{a}), 8$ and 9 , and the jets are the mechanisms whereby the protostars, or PMS stars, rid themselves of the otherwise large magnetic fields. For a more exhausted list of references, see those quoted below.

Acknowledgments. This work has been carried out under an operating grant from the Natural Sciences and Engineering Research Council of Canada. The National Radio Astronomy Observatory is a facility of the NSF, operated under a cooperative agreement by Associated Universities, Inc.

\section{References}

Cohen, R. J., Rowland, P. R., \& Blair, M. M. 1984. MNRAS, 210, 425-438.

Goetz, et al. 1995. BAAS, 27, 1318.

Hughes, V. A. 1985. ApJ, 298, 830-838.

Hughes, V. A. 1991. ApJ, 383, 280-288.

Hughes, V. A. 1993. $A J$, 105, 331-338.

Hughes, V. A. 1997. $A p J, 481,857-865$.

Hughes, V. A., \& Wouterloot, J. G. A. 1984. ApJ, 276, 204-210. 